\title{
Prorrenin/renin receptor expression in the solitary tract nucleus in a 5/6 nephrectomy-induced hypertension model in rats
}

\author{
Pedro Lopez-Sanchez ${ }^{1}$, Gabriela Arteaga-Bravo ${ }^{1}$, Ana V. Vega ${ }^{2}$, Yumei Feng ${ }^{3}$, Maria \\ Elena Hernandez-Campos ${ }^{1}$
}

${ }^{I}$ Seccion de Estudios de Posgrados e Investigacion, Escuela Superior de Medicina del IPN, Mexico, ${ }^{2} U B I M E D, F E S$ Iztacala-UNAM, Mexico, ${ }^{3}$ Center for Cardiovascular Research. School of Medicine. University of Nevada, USA

The interaction between the sympathetic nervous system (SNS) and the renin angiotensin system (RAS) is complex. The RAS has a component that is called the renin/prorenin receptor (PRR). The PRR was previously identified in several regions of the brain, such as the solitary tract nucleus (STN), which is part of the SNS, and participates in blood pressure regulation. Previous studies show that the alteration in the expression and function of PRR in this nucleus can trigger hypertension. However, if hypertension has another origin, it is not known how this receptor responds in this nucleus. The objective of the work was to determine the expression and activity of PRR in the solitary tract nucleus (STN) in rats with 5/6 nephrectomy-induced hypertension (NPX). Hypertension was induced using 5/6 nephrectomy, animals were subsequently sacrificed at seven and 15 days to extract the brain. Sagittal sections were made and immunofluorescence was performed to identify the expression of PRR and PLZF in the STN. The profile of gene expression in the brainstem was analyzed by q-PCR.

Our results showed the expression of PRR and PLZF in the STN by immunofluorescence. This expression was clear in control rats' neurons, and decreased significantly when the rats were NPX. PLZF in these same neurons also decreased significantly. There was no expression of PRR in glial cells. The immunofluorescence indicated that in sham rats, PLZF is translocated to the nucleus of the neuron, while in the NPX rats, it was located in the periphery of cells, which indicates a decrease in its activity within these cells, and therefore, PRR activity. The q-PCR reported a tendency to decrease in the expression of the PRR gene in the STN and the PLZF in the STN in NPX rats. An interesting finding was that in glial cells, PLZF increased significantly, but independently, of PRR.

Our results suggest that, in 5/6 nephrectomy, the PRR is inactivated in the STN, suggesting an effect of this receptor in the efferent sympathetic discharge; and that the PLZF seems to play a role in the glial function in this model. 